\title{
Laser Oscillation in Monolithic Molecular Single Crystals**
}

By Musubu Ichikawa,* Ryota Hibino, Masamitsu Inoue, Takeshi Haritani, Shu Hotta, Kei-ichi Araki, Toshiki Koyama and Yoshio Taniguchi

[*] Dr. M. Ichikawa, Mr. R. Hibino, Dr. M. Inoue, Mr. T. Haritani, Mr. K. Araki, Dr. T. Koyama, Prof. Y. Taniguchi

Department of Functional Polymer Science

Faculty of Textile Science and Technology

Shinshu University

Tokita 3-15-1, Ueda 386-8567 (Japan)

E-mail: musubu@giptc.shinshu-u.ac.jp

Prof. S. Hotta

Department of Polymer Science and Engineering

Faculty of Textile Science

Kyoto Institute of Technology,

Matsugasaki, Sakyo-ku, Kyoto 606-8585 (Japan)

[**] This work was supported by the Cooperative Link for Unique Science and Technology for Economy Revitalization (CLUSTER) of Japan's Ministry of Education, Culture, Sports, Science and Technology. It was also supported by the New Energy and Industrial Technology Development Organization (NEDO), in the area of Organic Materials Technology for Solid-State Injection Laser application, and by the Ministry's 21st Century COE program. 


\section{Text for Table of Content}

This Communication reports for the first time on the clear evidence about laser oscillation in monolithic molecular single crystals of a thiophene/phenylene co-oligomer (P6T). The laser oscillations are characterized by naturally formed crystal facet cavities with molecular-scale flatness. The multi-mode laser oscillation in a Fabry-Pérot resonator is characterized by sharply resolved spectral lines with their full width at half maximum down to only $38 \mathrm{pm}$. The laser oscillation is characterized by the presence of a well-defined threshold. 
The achievement of long-lasting, bright electroluminescence with high efficiency in both polymeric[Burroughes, 1990 \#276] and low-molecular-weight organic materials[Tang, 1989 \#27] has strongly motivated the development of a new class of laser diodes (LDs). Within this framework, optically induced solid-state organic lasers have been proposed and fabricated in the form of thin films by methods such as solution casting and vacuum deposition with a wide class of related organic semiconductors.[Tessler, 1996 \#256; Hide, 1996 \#237; McGehee, 2000 \#325; Kozlov, 1997 \#183; Bulovic, 1998 \#60] Prior to this recently developed research stream, laser oscillation in organic molecular single crystals was conceptually postulated by $\operatorname{Karl}[\operatorname{Karl}, 1972$ \#321] in the early 1970s. Although this led to the first demonstration of optically pumped lasing in "doped" molecular crystals,[Karl, 1972 \#321] to date, laser oscillation in a "monolithic" (undoped) single crystal has not yet been demonstrated. At the moment, however, there are several examples of stimulated emission including amplified spontaneous emission[Fichou, 1997 \#211; Garnier, 1998 \#312; Avanesjan, 1974 \#320; Brouwer, 1998 \#326; Yanagi, 2001 \#159; Nagawa, 2002 \#203] (ASE) from molecular single crystals. Here we show the first demonstration of optically induced laser oscillation in single crystals.

Laser emission from monolithic organic single crystals has proven highly problematic since Karl first suggested the possibility.[Karl, 1972 \#321] His reasoning was as follows. (i) As optical pumping usually creates mobile excitons in molecular crystals, these excitons may well collide with each other and dissipate their energy non-radiatively.[Bergman, 1967 \#324] (ii) Because nearly all molecules within a certain volume in a crystal have to be pumped to produce population inversion, this will result in high energy densities, producing serious thermal strain. A similar discussion has been given by Baldo et al.[Baldo, 2002 \#250] Nonetheless, molecular single crystals of $\pi$-conjugated oligomers have raised great interest in recent literature for their excellent, unique photonic properties, such as ASE[Fichou, 1997 \#211; Garnier, 1998 \#312; Avanesjan, 1974 \#320; Brouwer, 1998 \#326; Yanagi, 2001 \#159; Nagawa, 2002 \#203] 
and stimulated Raman scattering[Maksimov, 1981 \#313; Yanagi, 2003 \#295], based on cooperative interaction among the aligned molecules. Furthermore, monolithic organic molecular single crystals have the advantage of exhibiting superior charge-carrier transport properties as compared with amorphous and polymeric organic semiconductors.[note, \#268] This advantage results from the long-range structural ordering that these single crystals inherently possess. These excellent photonic and electronic features lead to the expectation that current-injection lasing will be achieved. Thus, demonstration of laser oscillation in a single crystal is a top priority.

In this Communication, we describe how we have solved the historic open question raised by Karl in regard to monolithic molecular crystals. We have definitively observed laser oscillation by optically pumping a thin-plate single crystal with parallel crystal faces on either end, which together function as a Fabry-Pérot cavity. The key point is that we have carefully fabricated the single crystals by using a new method of crystal growth.[Ichikawa, 2003 \#231] Most importantly, this method produces crystal faces, and thus, Fabry-Pérot cavities, of optically high flatness. This ensures strong self-cavity optical confinement in these crystals. We emphasize here that, unlike thin films, monolithic single crystals are hard to equip with a resonator without hampering its operation. Another key point lies in selecting a molecular semiconductor in which thiophenes and phenylenes are suitably hybridized at the molecular level.[Hotta, 2003 \#293] Since the crystallographic structure of the single crystals that we used has been fully determined,[Hotta, 2004 \#294] the relationship between that structure and the photo-pumping geometry can be uniquely defined.

We used crystals of a thiophene/phenylene co-oligomer, P6T[Hotta, 2003 \#293] (see Fig. 1a for the structural formula). The crystals were fabricated as thin rectangle plates (typically around 3-10 mm long, 0.5-1 mm wide, and 1-3 $\mu \mathrm{m}$ thick). Figures $1 \mathrm{~b}$ and 1c show micrographs of an as-grown single crystal of P6T at different magnifications. The thin crystals function as slab waveguides, ensuring that photons are confined and propagate within the slabs. The slab planes exhibit molecular-scale 
flatness (around a few nanometers within an area of $5 \mu \mathrm{m}$ x $5 \mu \mathrm{m}$ ) with steps of about 3 nm (approximately 1 step per 1-5 $\mu \mathrm{m}$ ), as confirmed by atomic force microscope (AFM) observations presented in Fig. 1d. This step size is equivalent to half the lattice constant of $6.188 \mathrm{~nm}$ for the $c$-axis of a P6T crystal (i.e., the length of a single molecule).[Hotta, 2004 \#294]

The features present in the scanning electron microscope (SEM) photograph in Fig. 1c are fully consistent with the crystallographic data for P6T.[Hotta, 2004 \#294] Note that the pair of crystal faces paralleling the ac-plane constitutes an optical resonator with its length defined as the separation between the two ends (measured along the $b$-axis). In the case of the sample shown in Fig. $1 b$ and designated as Sample A, for instance, the resonator length is $0.462 \mathrm{~mm}$. These crystal end faces cross the other pair of crystal faces (i.e., the $a b$-plane) at right angles. Thus, these two pairs of crystal faces with the molecular-scale flatness can provide strong, two-dimensional self-cavity optical confinement. From the single crystals that we grew, we carefully selected two more samples with different resonator lengths (Sample B: $0.560 \mathrm{~mm}$; Sample C: $1.02 \mathrm{~mm}$ ). The part of each crystal surrounded by the two pairs of faces was entirely photo-pumped to detect the photoemission and record its spectrum (see Fig. 1e for the schematic experimental setup).

Figure 2 shows the laser-induced emission spectrum of Sample A at the excitation (incident) laser fluence of $1 \mathrm{~mJ} / \mathrm{cm}^{2}$. The photoemission was highly directional and exhibited superior output stability after thousands of shots with the excitation pulse laser. As seen in the figure, a progression of extremely narrow emission lines clearly appears around $689 \mathrm{~nm}$. The full width at half maximum (FWHM) of the individual sharp lines is limited to only $38 \mathrm{pm}$ (as shown in the inset of Fig. 2), close to our experimental limit of $35 \mathrm{pm}$. These narrow lines arise regularly at an interval of $121 \mathrm{pm}$. A frequency power spectrum obtained with a fast Fourier transform of the emission spectrum (Fig. 2b) indicates that a major periodic peak occurs at a frequency of $8.30 \mathrm{~nm}^{-1}$, corresponding to the interval $121 \mathrm{pm}$. There are no other lower frequency peaks with 
intensities comparable to that peak.

Figure 3 shows photopumping intensity dependence of peak intensities of photoluminescence (PL) spectra from the same sample. A threshold is clearly noted at $\sim 750 \mu \mathrm{J} / \mathrm{cm}^{2}$. Concomitantly, the spectral profiles dramatically change below and above the threshold (compare Figs. 2a and the inset of Fig. 3). Below the threshold emission spectra are weak and featureless (see the inset of Fig. 3), reflecting a nature of a spontaneous emission. The strongly gain-narrowed and intensified spikes in Fig. 2a present a striking contrast to this emission. The spectra taken under a weak excitation imply that the center wavelength of the laser oscillation of $689 \mathrm{~nm}$ agrees with that of the second vibronic peak (assigned to the $0-1$ transition).

The aforementioned spectroscopic characteristics are reproducibly observed for two other samples (Samples B and C, see Fig. 4a and 4b). The mode intervals within a progression of a set of narrowed lines are 96 and $52 \mathrm{pm}$ for Samples B and C, respectively. Fourier analyses again indicate the presence of a major periodic peak in agreement with the above intervals. Figure 5 shows the resonator-length dependence of the mode interval for the three Samples A, B, and C. The diagram obviously indicates that the interval of the progressively structured emissions (see Figs 2, 4a, and $4 \mathrm{~b}$ ) is inversely proportional to the resonator length.

This is in good accordance with the well-known relationship between the mode intervals $(\Delta \lambda)$ and resonator lengths $(L)$ as approximated below[Yariv, $1989 \# 334$ ]

$$
\Delta \lambda \propto \lambda^{2} / 2 L,
$$

where $\lambda$ is the center wavelength of the laser oscillation $(\approx 689 \mathrm{~nm})$. The set of data in Fig. 5 is therefore a direct consequence of the presence of longitudinal cavity multi-modes confined as standing waves in the resonator. The Fourier analyses again confirm that additional modes (i.e. transverse modes) are of secondary importance. The highly directional emissions accompanied by the clear transition in the spectral profiles 
(Figs. 2a and 3) thus demonstrate that the relevant photoemissions result from the laser oscillation within the Fabry-Pérot cavities comprising the crystal faces on either end of the slab crystal.

In Figure 4c we depict for comparison the photoluminescence (PL) spectrum from a crystal in which the pertinent crystal faces are of lower quality (Sample D). This spectrum was otherwise taken under the same experimental conditions as the above three samples. It is relatively broad (FWHM: $3.7 \mathrm{~nm}$ ) and featureless and constitutes an envelope of the sharply resolved progressions of lines demonstrated in Figs. 2a, 4a, and $4 b$.

It is worth mentioning the polarization characteristics of these emissions in association with the crystal structure. The P6T molecules are aligned with the molecular long axis, tilted by $21^{\circ}$ from the normal to the $a b$-plane (the horizontal faces), and the direction of the transition dipole moment of these molecules is pretty close to that of the molecular long axis.[Hotta, 2004 \#294] Therefore, we anticipate that the output beam is preferentially polarized along this normal direction. Defining the index of the polarization as the intensity ratio of the light polarized parallel to the normal to that polarized perpendicular to it, we obtained a value of about 4 . This seems consistent with our expectation.

In conclusion, we have demonstrated, for the first time, the laser oscillation in self-cavity single crystals of P6T. The laser oscillation is characterized by the presence of a clear threshold with the photopumping intensities. The sharply resolved emission lines (with FWHM down to $38 \mathrm{pm}$ ) arise from the longitudinal cavity multi-modes with additional modes (i.e. transverse modes) of secondary importance. The essential point is that in a slab crystal of P6T, a pair of crystal faces corresponding to the ac-plane constitutes a perfect optical resonator (a Fabry-Pérot resonator), with its length defined as the separation between the two faces. The lasing occurs at the wavelength of the second vibronic peak of spontaneous emission from the P6T crystals. 


\section{Experimental}

The synthesis and purification methods for P6T can be found elsewhere.[Hotta, 2003 \#293] The crystal growth procedure is described in our previous report.[Ichikawa, 2003 \#231] The SEM photograph was taken with a Hitachi S-5000 field emission scanning electron microscope at a tilt angle of $40^{\circ}$ against the normal of the slab crystal plane. The AFM observations were carried out in the dynamic mode using a Shimadzu SPM-9500J3 scanning probe microscope. An $\mathrm{N}_{2}$-gas laser, generating a light pulse of 500 -ps duration at $337.1 \mathrm{~nm}$ with a repetition rate of $10 \mathrm{~Hz}$, was used as the excitation light source. The rectangular-focused $(1 \mathrm{~mm} \times 5 \mathrm{~mm})$ excitation laser light was normally incident to a horizontal crystal face that adhered closely to a quartz substrate. The light emitted from the end face was normally detected against the end face through a UV-cut filter by using a fiber bundle attached spectrograph (Solar TII, MS7504) with a charge-coupled device camera (Andor, DV420-OE). The solid angle of the light capturing system was 0.04 sr. Fourier analysis was performed on commercial software (Origin Lab., Origin 7) with a rectangular window function.

\section{References and Notes}

\section{Figure Captions}

Figure 1 Details of the P6T crystal lasers. a, Chemical structure of P6T. b, Global image of a P6T single crystal. The right inset shows the crystallographic structure of P6T. c, Scanning electron microscope image of a P6T crystal. The crystal face corresponding to the $a c$-plane is represented as a vertical "cliff". The $a b$-plane parallels the substrate plane. d, AFM image of the surface ( $a b$-plane) of a P6T crystal. The surface profile (represented with a notched white line) taken along the line $\mathrm{A}-\mathrm{B}$ clearly indicates the molecularly resolved steps. e, Schematic of the laser structure with the P6T crystal and our experimental setup. The crystal face shown in black-hatched 
yellow corresponds to the cliff face shown in c, and together with the face on the opposite side, it constitutes a Fabry-Pérot cavity.

Figure 2 a: Laser-induced light emission spectrum of a P6T crystal (Sample A) at an incident laser fluence of $1 \mathrm{~mJ} / \mathrm{cm}^{2}$. The resonator length, defined as the separation between the two end faces (corresponding to the crystallographic ac-plane), is 0.462 $\mathrm{mm}$. The inset shows an enlarged profile from the same spectrum. b: Frequency power spectrum obtained with a fast Fourier transform of the emission spectrum.

Figure 3 Photopumping intensity dependence of peak intensities of PL spectra from the P6T crystal (Sample A). The data are indicated with closed circles. The inset depicts a spectrum taken with a fluence of $570 \mu \mathrm{J} / \mathrm{cm}^{2}$.

Figure 4 Laser-induced light emission spectra of three P6T crystals at an incident laser fluence of $1 \mathrm{~mJ} / \mathrm{cm}^{2}$. The resonator lengths of the crystals are a: 0.560 mm (Sample B) and b: $1.03 \mathrm{~mm}$ (Sample C). c: No cavity (Sample D, the pertinent end faces of this crystal have insufficient quality).

Figure 5 Resonator-length dependence of the mode interval for Samples A, B, and $\mathrm{C}$. The solid line was obtained by least mean square fitting with a constraint of the origin $(0,0)$. 


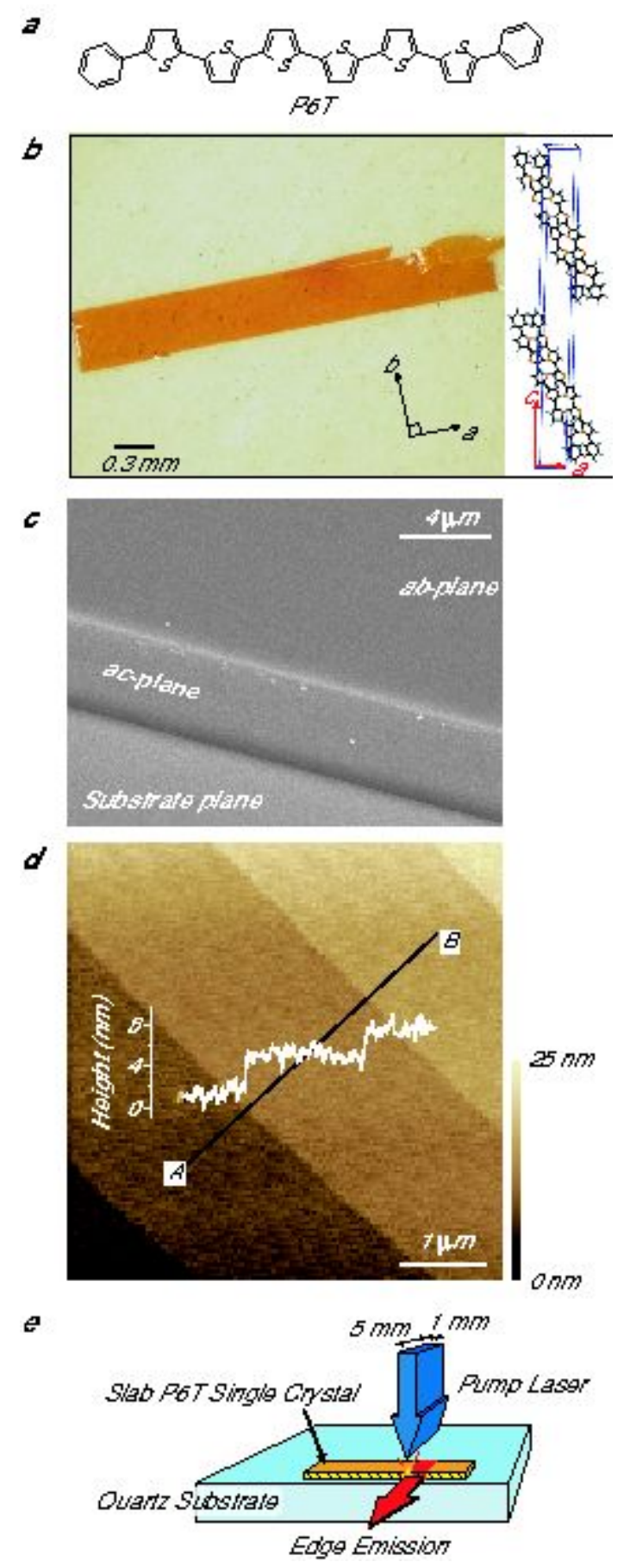

Fig. 1 

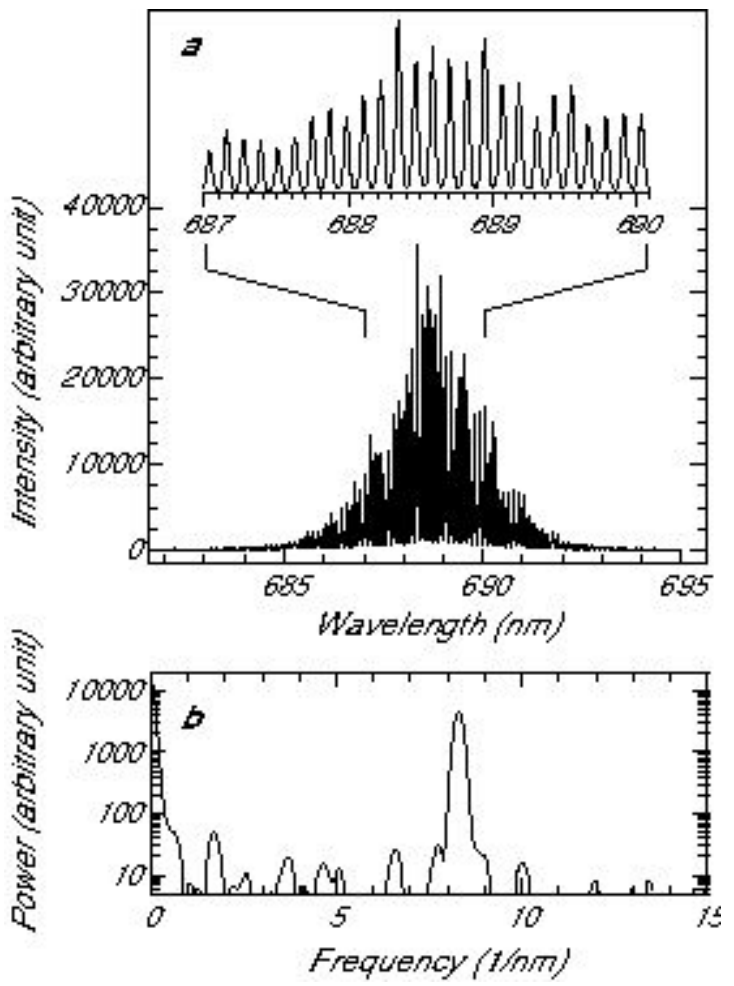

Fig. 2

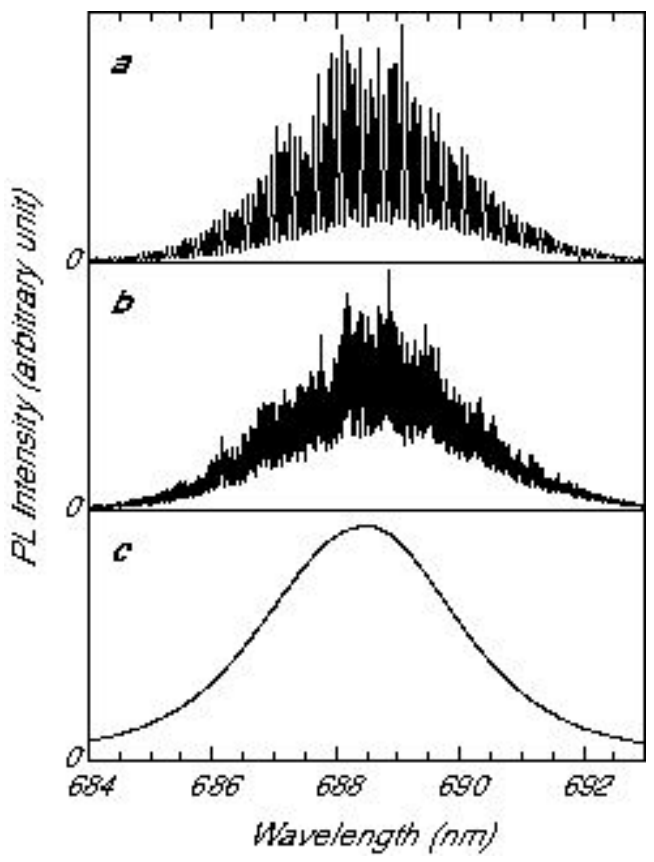

Fig. 4

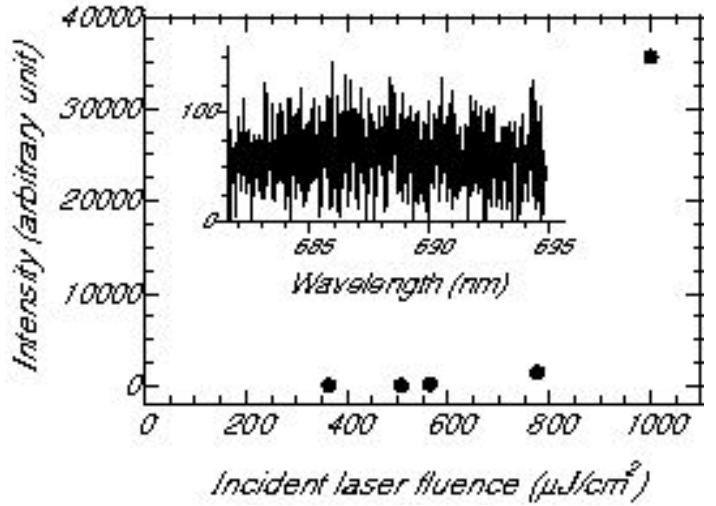

Fig. 3

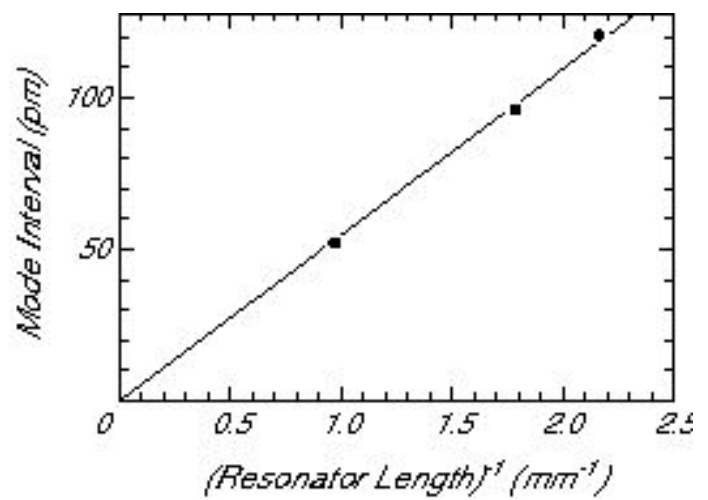

Fig. 5 